\title{
Genes que expresan resistencia a carbapenemasas presentes en Pseudomona aeruginosa
}

\section{Genes expressing carbapenemase resistance present in Pseudomona aeruginosa}

José Enrique Oliva-Menacho 1,a

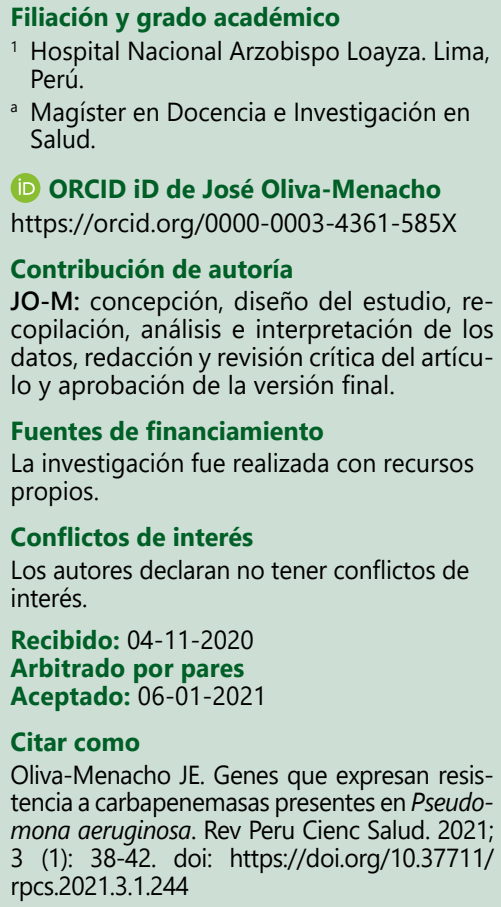

\section{RESUMEN}

Objetivo. Describir los genes que expresan resistencia a la carbapenemasas presentes en la Pseudomona aeruginosa. Métodos. Se realizó la revisión de la literatura científica utilizando el método PRISMA, durante febrero de 2020, en las bases de datos Pubmed, Scopus, y Scielo. Resultados. Se describe la reacción en cadena de la polimerasa (PCR) como la técnica más utilizada; además, se hallaron los genes blancos moleculares de hiperexpresión de múltiples bombas de expulsión que generan la resistencia bacteriana. Conclusiones. La PCR convencional y multiplex es la técnica más utilizada para el diagnóstico de genes que expresan resistencia a carbapenemasas presentes en Pseudomona aeruginosa de pacientes con fibrosis quística, al ser la más estandarizada y tener alta sensibilidad, y especificidad, habiéndose detectado asimismo genes IMP, VIM, OXA, MexAB-OprM, MexCD-OprJ, MexEF-OprN y MexXY-OprM.

Palabras clave: fibrosis quística; carbapenémicas; técnicas moleculares; detección; PCR; Perú (Fuente: DeCS - BIREME).

\section{ABSTRACT}

Objective. To describe the genes expressing carbapenemase resistance present in Pseudomona aeruginosa. Methods. The scientific literature was reviewed using the PRISMA method during February 2020 in the Pubmed, Scopus and Scielo databases. Results. The polymerase chain reaction (PCR) is described as the most commonly used technique; in addition, molecular white hyperexpression genes of multiple ejection pumps that generate bacterial resistance were found. Conclusions. Conventional and multiplex PCR is the most widely used technique for the diagnosis of genes that express resistance to carbapenemases present in Pseudomona aeruginosa of patients with cystic fibrosis, being the most standardized and have high sensitivity, and specificity, also having detected IMP, VIM, OXA, MexAB-OprM, MexCDOprJ, MexEF-OprN and MexXY-OprM genes.

Keywords: cystic fibrosis; carbapenemics; molecular techniques; detection; $P C R$; Peru (Source: MeSH-NLM). 


\section{INTRODUCCIÓN}

La Pseudomonas aeruginosa es un agente causal de infecciones intrahospitalarias que produce bronconeumonía difusa bilateral en el aparato respiratorio con formación de microabscesos y necrosis tisular ${ }^{(1)}$.

La fibrosis quística es el defecto genético debido a mutaciones en un gen del brazo largo del cromosoma 7 que codifica al regulador de la conductancia transmembrana de la fibrosis quística (CFTR). Este regulador interviene en el balance de fluidos a través de las células epiteliales, actúa como un canal para el pase del cloro e inhibe la absorción de sodio ${ }^{(2)}$. La infección pulmonar es, a su vez, una de las causas más importantes de morbilidad y mortalidad en paciente de fibrosis quística ${ }^{(3)}$.

La enfermedad broncopulmonar es la causa de muerte en el $95 \%$ de los pacientes con fibrosis quística. Los organismos bacterianos más frecuentemente aislados son: Pseudomona aeruginosa, Staphylococcus aureus y Haemophilus influenzae ${ }^{(4)}$.El Staphylococcus aureus es usualmente el primer patógeno aislado de la vía aérea de los pacientes con fibrosis quística, con una tasa de infección que disminuye con la edad, mientras que, por su parte, la tasa de Pseudomona aeruginosa se incrementa. La presencia de Pseudomona aeruginosa en el tracto respiratorio en forma intermitente obliga a su erradicación completa por ser el agente que mayor destrucción produce en las vías aéreas. A través de la presencia primaria del Staphylococcus aureus en el tracto respiratorio en forma intermitente, la Pseudomona aeruginosa ve facilitada su entrada y posterior colonización. Si bien ambas bacterias pueden coexistir, el desarrollo abundante de Pseudomona aeruginosa puede inhibir el crecimiento de staphylococcus aureus ${ }^{(5-7)}$.

La Pseudomona aeruginosa es el primer causante de neumonía asociada a la ventilación mecánica (NAV) en las unidades de cuidados intensivos (UCI), con tasas de mortalidad del $50 \%{ }^{(6,8,9)}$. Por consiguiente, el objetivo del presente estudio fue describir los genes que expresan resistencia a las carbapenemasas presentes en la pseudomona aeruginosa.

\section{MÉTODOS}

Se realizó la revisión de literatura científica utilizando el método PRISMA, durante febrero de 2020, en las bases de datos Pubmed, Science Direct, Elsevier y Scielo; como criterios de inclusión artículos originales publicados entre los años 1988 y 2020 que basados en investigación de secuenciamientos moleculares de carbapenemasas presentes en pseudomona aeruginosa.

\section{RESULTADOS}

La Pseudomona aeruginosa es el principal causante de infección pulmonar crónica en pacientes con fibrosis quística (FQ), enfermedad pulmonar obstructiva crónica (EPOC) y bronquiectasias ${ }^{(10,11)}$. Una de las características más preocupantes es su elevado nivel de resistencia intrínseca a los antibióticos, así como su gran capacidad de desarrollo de nuevas resistencias a través de mutaciones en su cromosoma. En este sentido, cabe destacar las múltiples mutaciones en ampR, ampD o dacB (PBP4) ${ }^{(12,13)}$ que determinan la hiperproducción de la betalactamasa cromosómica tipo AmpC, confiriendo resistencia a todas las penicilinas (incluyendo las combinaciones con inhibidores de betalactamasas, como la piperacilinatazobactam), cefalosporinas (ceftazidima y cefepima) y monobactámicos (aztreonam).

Es característica también de pseudomona aeruginosa la resistencia mediada por la hiperexpresión de alguna de las múltiples bombas de expulsión codificadas en su genoma: MexAB-OprM, MexCD-OprJ, MexEF-OprN y MexXY-OprM, principalmente; las cuales, dependiendo de la bomba implicada, pueden afectar a prácticamente todos los betalactámicos, fluoroquinolonas y aminoglucósidos. Por último, cabe destacar también la represión o inactivación de la porina OprD, que confiere resistencia a las carbapenemasas ${ }^{(14,15)}$ y la distribución de genes que expresan resistencia (ver tabla 1).

Tabla 1. Genes que expresan mecanismos de resistencia por Pseudomona aeruginosa

\begin{tabular}{|c|c|c|}
\hline Mecanismo & Gen /Proteína & $\begin{array}{c}\text { Resistencia al } \\
\text { antimicrobiano }\end{array}$ \\
\hline $\begin{array}{l}\text { Impermeabilidad } \\
\text { de la membrana } \\
\text { externa }\end{array}$ & OprD,OprF,OprB & $\begin{array}{c}\text { Imipenem, meropenem, } \\
\text { quinolonas, } \\
\text { aminoglucósidos }\end{array}$ \\
\hline Bombas de eflujo & $\begin{array}{l}\text { MexAB-OprM, MexXY- } \\
\text { OprM, MexCD-OprJ, } \\
\text { MexEF-OprN }\end{array}$ & $\begin{array}{l}\text { } \text {-lactámicos, } \\
\text { fluoroquinolonas, } \\
\text { aminoglucósidos }\end{array}$ \\
\hline$\beta$-Lactamasas & $\begin{array}{c}\text { AmpD, DacB, AmpR, } \\
\text { AmpC }\end{array}$ & $\begin{array}{l}\beta \text {-lactámicos, penicilinas } \\
\text { antipseudomonas }\end{array}$ \\
\hline $\begin{array}{l}\text { Modificación } \\
\text { del blanco del } \\
\text { antimicrobiano }\end{array}$ & $\begin{array}{c}\text { GyrA7,GyrB, } \\
\text { topoisomerasa tipolV } \\
\text { (ParC/ParE) rmtA }\end{array}$ & $\begin{array}{l}\text { Fluoroquinolonas, amino- } \\
\text { glucósidos }\end{array}$ \\
\hline $\begin{array}{l}\text { Transferencia } \\
\text { horizontal }\end{array}$ & $\begin{array}{c}\text { Metalo- } \beta \text {-lactamasas- } \\
\text { clase B } \\
\text { Genes codificantes de } \\
\text { MBLs: } \\
\text { bla VIM-1 y bla VIM-2 }\end{array}$ & $\begin{array}{c}\text { Penicilinas, } \\
\text { cefalosporinas, imipenem } \\
\text { Las MBLs no hidrolizan } \\
\text { Aztreonam } \\
\text { AZT: S / IMl ó MER: R } \\
\text { probable MBL }\end{array}$ \\
\hline $\begin{array}{l}\text { Cambios en la } \\
\text { membrana }\end{array}$ & $\begin{array}{l}\text { Modificación lípido } \\
\text { A Sobreexpresión de } \\
\text { AmpC }\end{array}$ & Penicilinas \\
\hline
\end{tabular}


El problema se ve aumentado en las cepas causantes de infección respiratoria crónica, a menudo asociadas con elevadas tasas de mutación y, por lo tanto, de desarrollo de resistencias ${ }^{(16,17)}$, así como con la formación de biopelículas en el pulmón, lo que limita drásticamente la actividad de los antibióticos ${ }^{(14)}$. Además, la Pseudomona aeruginosa es capaz de adquirir nuevos determinantes de resistencia por transferencia horizontal. Entre los determinantes adquiridos, normalmente en forma de casetes situados en integrones, a su vez localizados en transposones o plásmidos que permiten su movilidad, destacan las betalactamasas, incluyendo las de espectro extendido (BLEE) y las carbapenemasas, pero también las enzimas modificantes de los aminoglucósidos ${ }^{(18)}$.

La resistencia a las carbapenemas en la pseudomona aeruginosa puede tener un origen cromosómico, por mutaciones en determinados genes, o bien estar mediada por la adquisición horizontal de carbapenemasas ${ }^{(19-21)}$. Entre los mecanismos mutacionales más conocidos destaca la represión o inactivación de la porina OprD, por la que se introducen las carbapenemasas en la célula bacteriana, que confiere resistencia al imipenem y sensibilidad reducida al meropenem. Para la adquisición de resistencia de alto nivel a esta última carbapenema, y también al recientemente introducido doripenem ${ }^{(20)}$, la Pseudomona aeruginosa necesita, además, acumular mutaciones causantes de hiperproducción de su betalactamasa cromosómica $\mathrm{AmpC}$, que se pueden dar en diferentes genes reguladores, como ampR, ampD o dacB ${ }^{(22,23)}$ o en mutaciones que conduzcan a la hiperexpresión de la bomba de expulsión MexAB-OprM ${ }^{(18,19,21)}$. Estas mutaciones pueden afectar al gen represor de la bomba, mexR, o bien a otros reguladores secundarios: nalC y naID. Por otra parte, si bien la bomba MexEF-OprN no expulsa de forma significativa las carbapenemas, su hiperexpresión se relaciona con la pérdida de sensibilidad a estos antibióticos. El mecanismo por el cual se produce este hecho no es del todo conocido, pero parece deberse a que las mutaciones que determinan la hiperexpresión de la bomba, en el gen regulador mexT, también conllevan la reducción de la expresión de OprD ${ }^{(19,20,21,22)}$.

Sin duda, uno de los principales problemas emergentes, tanto en enterobacterias como en gramnegativos no fermentadores, incluyendo, obviamente, pseudomonas auriginosa es la creciente diseminación de las carbapenemasas transferibles. Éstas pertenecen a tres de las cuatro clases estructurales definidas por Ambler ${ }^{(23)}$ : $A$ y $D$, ambas con serina en el centro activo, y $B$ (metalo- $\beta$ lactamasas [MBL], que necesitan zinc en su centro activo para ser funcionales) ${ }^{(24)}$. Por otra parte, recientemente se ha propuesto la existencia de betalactamasas tipo
AmpC cromosómicas de espectro extendido (ESAC) en Pseudomona aeruginosa definidas por la presencia del polimorfismo T105A, que determina una cierta actividad carbapenemasas y que, por lo tanto, puede contribuir a la resistencia a estos antibióticos ${ }^{(25)}$.

\section{DISCUSIÓN}

La utilización de técnicas de biología molecular en las muestras microbiológicas de los pacientes con fibrosis quística permite: a) detectar microorganismos de forma directa en las secreciones respiratorias sin necesidad de tener que esperar al cultivo microbiológico $\left.{ }^{(26)} ; b\right)$ detectar microorganismos de crecimiento difícil, cuyo cultivo con las técnicas habituales se produce con escasa o nula rentabilidad, o en casos de bajos recuentos o colonizaciones mixtas; c) identificar patógenos con taxonomía incierta ${ }^{(27)}$; d) documentar la persistencia y variabilidad de la clonalidad en un mismo paciente así como de posibles infecciones cruzadas entre distintos pacientes ${ }^{(28)} y$; e) facilitar el seguimiento del tratamiento antimicrobiano y control de la erradicación.

La detección de microorganismos de forma directa en las muestras respiratorias, sin necesidad de tener que esperar al cultivo microbiológico, ha permitido detectar la presencia de pseudomona aeruginosa en los periodos ventana (en los que, debido al tratamiento antimicrobiano, pueden disminuir los recuentos) y encontrarse por debajo del límite de detección del cultivo y también en los periodos iniciales (en los que su bajo número escapa a su detección con el cultivo). Las técnicas de biología molecular han permitido demostrar que la colonización por Pseudomona aeruginosa y Stenotrophomonas maltophilia persiste aun cuando los cultivos son negativos, siendo de gran ayuda en la valoración del tratamiento antimicrobiano ${ }^{(29)}$.

En la infección inicial se recomienda tratamiento inhalado 28 días con colistina $(0,5-2 \mathrm{MU} / 8 \mathrm{~h})$, tobramicina $(300 \mathrm{mg} / 12 \mathrm{~h})$ o aztreonam $(75 \mathrm{mg} / 8 \mathrm{~h})$ con o sin ciprofloxacino oral (15-20mg/kg/12h, 2-3 semanas). En la infección crónica se recomienda solo vía inhalada en tratamiento continuo con colistina, o en ciclos onoff de 28 días con tobramicina o aztreonam. Durante las exacerbaciones leves moderadas se recomienda tratamiento oral (ciprofloxacino, 2-3 semanas) y en las graves, tratamiento intravenoso ( $\beta$-lactámico asociado a un aminoglucósido o una fluoroquinolona) ${ }^{(30)}$.

En conclusión, las metalo- $\beta$-lactamasas son las carbapenemasas de mayor importancia clínica y epidemiológica a nivel mundial, debido al alto nivel 
de resistencia que presentan para los $\beta$-lactámicos (excepto aztreonam) y la alta tasa de mortalidad asociada a la persistencia en el ambiente hospitalario. Algunos mecanismos de resistencia adicionales a los enzimáticos incluyen a las bombas de expulsión que se encuentran en la membrana externa de la célula y expulsan hacia el exterior de la bacteria gran cantidad de moléculas (metabolitos, detergentes, solventes orgánicos y antibióticos). El principal papel de este mecanismo es bajar las concentraciones de sustancias tóxicas dentro de la célula.

Teniendo en cuenta todo lo anteriormente mencionado, la investigación permitió conocer la magnitud del problema de carbapenemasas en pseudomona aeruginosa más importantes como patógenos nosocomiales, los tipos de carbapenemasas presentes en nuestro medio; una información con la que no contábamos por la carencia de estudios de este tipo. Por lo tanto, la información generada por el presente estudio contribuirá no solo a mejorar el abordaje terapéutico de las infecciones causadas por estos patógenos; lo más importante es que además puede constituirse como una herramienta valiosa para los estamentos correspondientes en la implementación de políticas de control de resistencia a antimicrobianos.

\section{REFERENCIAS}

1. Murray P, Kobayashi G, Pfaller M, Rosenthal K. Laboratory diagnosis of bacterial diseases. Pseudomonas and related non-fermenting bacteria. Medical Microbiology. 2a ed. Madrid: Harcourt Brace; 1997.

2. Ramsey BW. Management of pulmonary disease in patients with cystic fibrosis. New Engl J Med. 1996; 335(3):179-187. doi: 10.1056/NEJM199607183350307

3. Cantón R, Cobos N, De Gracia J, Baquero F, Honorato J, Gartner S, Álvarez A, Salcedo A, Oliver A, Gracia-Quetglas E, Spanish Consensus Group for Antimicrobial Therapy in the Cystic Fibrosis Patient. Antimicrobial treatment against lung colonization by pseudomonas aeruginosa in the patient with cystic fibrosis. Arch Bronconeumol. 2005; 41 Suppl 1: 1-25. doi: 10.1111/j.14690691.2005.01217.x

4. Campos M, Navarro R, Amuy E, Vargas A, Herrera Marco $L$. Bacterial isolates from the respiratory tract of children with cystic fibrosis of the pancreas. Rev méd Hosp Nac Niños (Costa Rica). 2000; 35 (1-2): 13-19.

5. Coutinho HDM, Falcão-Silva V, Fernandes Gonçalves G. Pulmonary bacterial agents and antibiotic therapy in patients with cystic fibrosis: a guide for healthcare personnel. International Archives of Medicine. 2008; 1(1): 1-7. doi: 10.1186/1755-7682-1-24

6. Pollack M. Pseudomonas aeruginosa. En: Mandell GL, Bennett JE, Dolin R, eds. Principles and practice of infectious diseases. 5a ed. Nueva York: Churchill Livingstone; 2000. p. 1980-2003.

7. Aloush V, Navon-Venezia S, Seigman-Igra Y, Cabili S, Carmeli Y. Multidrug-resistant Pseudomonas aeruginosa: risk factors and clinical impact. Antimicrob Agents Chemother. 2006; 50(1):43-48. doi: 10.1128/AAC.50.1.4348.2006

8. Bergmans DC, Bonten MJ, Van Tiel FH, Gaillard CA, van der Geest S, Wilting RM, et al. Cross-colonisation with pseudomonas aeruginosa of patients in an intensive care unit. Thorax. 1998; 53(12):1053-1058. doi:10.1136/thx.53.12.1053

9. Lynch JP. Hospital-acquired pneumonia: risk factors, microbiology, and treatment. Chest. 2001; 119(2 Suppl): 373S-384S. doi: 10.1378/chest.119.2_suppl.373s.

10. Gilligan PH. Microbiology of airway disease in patients with cystic fibrosis. Clin Microbiol Rev.1991; 4(1): 35-51. doi: 10.1128/cmr.4.1.35

11. Martinez-Solano L, Macia MD, Fajardo A, Oliver A, Martinez JL. Chronic Pseudomonas aeruginosa infection in chronic obstructive pulmonary disease. Clin Infect Dis. 2008; 47(12): 1526-1533. doi: 10.1086/593186

12. Juan $C$, Macia MD, Gutiérrez $O$, Vidal $C$, Pérez JL, Oliver A. Molecular mechanisms of beta-lactam resistance mediated by AmpC hyperproduction in Pseudomonas aeruginosa clinical strains. Antimicrob Agents Chemother. 2005; 49(11): 4733-4738. doi: 10.1128/AAC.49.11.47334738.2005

13. Moya B, Dötsch A, Juan C, Blázquez J, Zamorano L, Haussler S, Oliver A. Beta-lactam resistance response triggered by inactivation of a non-essential penicillin-binding protein. PLoS Pathog. 2009; 5(3).1-10. doi: 10.1371/ journal.ppat.1000353

14. Pedersen SS. Lung infection with alginate-producing, mucoid Pseudomonas aeruginosa in cystic fibrosis. APMIS Suppl. 1992; 28: 1-79.

15. Souza- Días MB, Habert AB, Borrasca V, Stempliuk V, Ciolli $A$, Araujo MR, et al. Salvage of long-term central venous catheters during an outbreak of Pseudomonas putida and Stenotrophomonas maltophilia infections associated with contaminated heparin catheter-lock solution. Infect Control Hosp Epidemiol. 2008; 29(2): 125-30. doi: $10.1086 / 526440$

16. Macia M, Blanquer D, Togores B, Sauleda J, Pérez JL, Oliver $A$. Hypermutation is a key factor in development of multiple-antimicrobial resistance in Pseudomonas aeruginosa strains causing chronic lung infections. Antimicrob Agents Chemother. 2005;49(8):3382- 3386. doi: 10.1128/AAC.49.8.3382-3386.2005

17. Oliver A, Cantón R, Campo P, Baquero F, Blazquez J. High frequency of hypermutable Pseudomonas aeruginosa in cystic fibrosis lung infection. Science. 2000; 288(5469): 1251-1254.doi: 10.1126/science.288.5469.1251

18. Mesaros N, Nordmann P, Plesiat P, Roussel-Delvallez M, Van Eldere J, Glupczynski Y, et al. Pseudomonas aeruginosa: resistance and therapeutics options in the turn of the new millennium. Clin Microbiol Infect. 2007; 13(6): 560-578. doi: 10.1111/j.1469-0691.2007.01681.x

19. Quale J, Bratu S, Gupta J, Landman D. Interplay of efflux system, ampC, and oprD expression in carbapenem resistance of Pseudomonas aeruginosa clinical isolates. Antimicrob Agents Chemother. 2006; 50(5): 1633-1641. doi: 10.1128/AAC.50.5.1633-1641. 2006

20. Llanes C, Hocquet D, Vogne C, Benali-Baitich D, Neuwirth C, Plesiat P. Clinical strains of Pseudomonas aeruginosa overproducing MexAB-OprM and MexXY efflux pumps simultaneously. Antimicrob Agents Chemother. 2004; 48(5):1797-1802. doi: 10.1128/aac.48.5.1797-1802.2004 
21. Lodge JM, Minchin SD, Piddock LJ, Busby JW. Cloning, sequencing, and analysis of the structural gene and regulatory region of the Pseudomonas aeruginosa chromosomal ampC -lactamase. J Biochem. 1990; 272: 627631. https://doi.org/10.1042/bj2720627

22. Maseda H, Sawada I, Saito K, Uchiyama H, Nakae T, Nomura N. Enhancement of the mexAB-oprM efflux pump expression by a quorum- sensing autoinducer and its cancellation by a regulator, MexT, of the mexEF-oprN efflux pump operon in Pseudomonas aeruginosa. Antimicrob Agents Chemother. 2004; 48(4): 1320 -1328. doi: 10.1128/aac.48.4.1320-1328.2004.

23. Ambler RP. The structure of beta-lactamases. Philos Trans R Soc Lond B Biol Sci.1980; 289(1036): 321-331. doi: 10.1098/rstb.1980.0049

24. Walsh TR, Toleman MA, Poirel L, Nordmann P. Metallo-beta-lactamases: the quiet before the storm? Clin Microbiol Rev. 2005; 18(2): 306-325. doi: 10.1128/CMR.18.2.306325.2005

25. Rodriguez-Martinez JM, Poirel L, Nordmann P. Molecular epidemiology and mechanisms of carbapenem resistance in Pseudomonas aeruginosa. Antimicrob Agents Chemother. 2009; 53 (11): 4783-4788. doi: 10.1128/ AAC.00574-09.

26. Xu J, Moore JE, Murphy PG. Millar B, Elborn J. Early detection of Pseudomonas aeruginosa. Comparison of conventional versus molecular (PCR) detection directly from adult patients with cystic fibrosis (CF). Ann Clin Microbiol Antimicrob. 2004; 20;3(1): 1-5. doi: 10.1186/14760711-3-21

27. Ferroni A, Sermet-Gaudelus I, Abachin E, Quesne G, Lenoir $G$, Berche $P$, et al. Use of $16 \mathrm{~S}$ rRNA gene sequencing for identification of nonfermenting gram-negative baciIli recovered from patients attending a single cystic fibrosis center. J Clin Microbiol. 2002; 40(10): 3793-3797. doi: 10.1128/JCM.40.10.3793-3797.2002

28. Anzai Y, Kim H, Park J, Wakabayashi H, Oyaizu. Phylogenetic affiliation of the pseudomonads based on $16 S$ rRNA sequence. Int J Syst Evol Microbiol. 2000; 50(4):1563-1589. doi: 10.1099/00207713-50-4-1563

29. LiPuma JJ, Dulaney BJ, McMenamin JD, Whitby PW, Stull TL, Coenye T, Vandamme P. Development of rRNA-based PCR assays for identification of Burkholderia cepacia complex isolates recovered from cystic fibrosis patients. J Clin Microbiol. 1999; 37(10): 3167-3170. doi:10.1128/ JCM.37.10.3167-3170.1999

30. Cantóna R, Máiz L, Escribanod A, Olveirae C, Oliverb A, Asensiog $\mathrm{O}$, et al. Spanish consensus for the prevention and treatment of bronchial infection by Pseudomonas aeruginosa in patients with cystic fibrosis. Arch Bronconeumol. 2015; 51(3): 140-150. doi: 10.1016/j.arbres.2014.09.021 